\title{
TERRESTRIAL SNAILS (MOLLUSCA: GASTROPODA) OF ISLANDS OF ŠOLTA, DRVENIK VELI AND DRVENIK MALI (CROATIA)
}

\author{
Vesna Štamol ${ }^{1}$, Zoltán Péter Erốss ${ }^{2}$, Eduard KletečKi ${ }^{1}$ \\ \& Marijana Vuković ${ }^{1}$
}

${ }^{1}$ Croatian Natural History Museum, Demetrova 1, 10000 Zagreb, Croatia ${ }^{2}$ Hungarian Natural History Museum, Baross 13, 1088 Budapest, Hungary

\begin{abstract}
Štamol, V., Erőss, Z.P., Kletečki, E. \& Vuković, M.: Terrestrial snails (Mollusca: Gastropoda) of islands of Šolta, Drvenik veli and Drvenik mali (Croatia). Nat. Croat., Vol. 26, No. 1., 45-64, Zagreb, 2017.

During the field work of our research into the terrestrial malacofauna of the eastern Adriatic islands Šolta, Drvenik veli and Drvenik mali (Croatia) 36 snail species with shells were registered. That almost tripled the number of taxa recorded in the literature. We consider Lindholmiola corcyrensis (Rossmässler, 1836) and Delima (Piceata) piceata (Rossmässler, 1836), registered in the literature for Drvenik veli, non-existing for the researched area, as well as for Croatia. Of the recorded taxa we particularly refer to Odontocyclas kokeilii (Rossmässler, 1837) with Šolta and Drvenik veli being its second i.e. third island localities in Croatia, and the endemic clausilid snail Delima (Semirugata) hiltrudis H. Nordsieck, 1969, also found on Šolta and so far known only from the island of Brač. The greatest number of species were found on the biggest island of Šolta, and the smallest island Drvenik mali had the lowest number of species, as expected. The values of the Whittaker index of biodiversity and of the maximum index of biodiversity increased according to size of island. The average number of taxa per site is the highest on the mid-sized island of Drvenik veli, which is a consequence of the diversity and good state of preservation of its habitats.
\end{abstract}

Key words: land snails, Adriatic islands, Šolta, Drvenik veli, Drvenik mali, Croatia.

Štamol, V., Erőss, Z.P., Kletečki, E. \& Vuković, M.: Kopneni puževi (Mollusca: Gastropoda) otoka Šolte, Drvenika velog i Drvenika malog (Hrvatska). Nat. Croat., Vol. 26, No. 1., 45-64, Zagreb, 2017.

Terenskim istraživanjem kopnene malakofaune istočnojadranskih otoka Šolte, Drvenika velog i Drvenika malog (Hrvatska) nađeno je 36 vrsta puževa s kućicom. Time smo gotovo utrostručili broj svojti zabilježenih u literaturi. Vrste Lindholmiola corcyrensis (Rossmässler, 1836) i Delima (Piceata) piceata (Rossmässler, 1836), zabilježene u literaturi za Drvenik veli, smatramo nepostojećim na istraživanom području, a i u Hrvatskoj. Od nađenih svojti ističemo Odontocyclas kokeilii (Rossmässler, 1837) koja na Solti i Drveniku velom ostvaruje drugo, odnosno treće otočno nalazište u Hrvatskoj, te endemičnu zaklopnicu Delima (Semirugata) hiltrudis H. Nordsieck, 1969, također nađenu na Šolti, a dosada poznatu samo s otoka Brača. Očekivano, najviše vrsta ima na najvećem otoku, Šolti, a najmanje na najmanjem otoku, Drveniku malom. Vrijednosti Whittakerovog indeksa biodiverziteta i maksimalnog indeksa biodiverziteta rastu s veličinom istraživanih otoka. Prosječan broj svojti po lokalitetu najveći je na srednje velikom istraživanom otoku Drveniku velom, što je posljedica raznolikosti staništa, i prvenstveno njihove očuvanosti.

Ključne riječi: kopneni puževi, jadranski otoci, Šolta, Drvenik veli, Drvenik mali, Hrvatska

\section{INTRODUCTION}

The eastern Adriatic coast of Croatia is one of the most indented and island-rich parts of the Mediterranean. There are 1244 above-sea terrestrial units: islands, islets and rocks 
and rocks awash (Duplančıć et al., 2004). These units are often characterized by distinctive flora and fauna, and threats and conservation needs different to those on the neighbouring mainland. The foundation for protection is knowing their plant and animal composition. Unfortunately for only ca 100 of them, which does not make up even $10 \%$, of published results of systematic research into land snails exist (Bole, 1958; ŠTAMOL, 1986, 2013; Štamol \& VelKovrh, 1995; Štamol \& Poje, 1998; ReischüTz \& Reischütz, 1999, 2000; Fischer et al., 2000; ReIschütz et al., 2002; ŠTAMOL \& KLETEČKI, 2005; ŠTAMOL et al., 2012). This paper gives for the first time results of systematic research into the land malacofauna of the central Dalmatian islands of Šolta, Drvenik veli and Drvenik mali, which has enabled their fauna and biodiversity to be presented and guidelines for their protection to be drawn up.

\section{STUDY AREA}

Šolta is a central Dalmatian island (Croatia) situated south of the coastal cities of Trogir and Split, west of the island of Brač and southeast of the islands of Drvenik mali and Drvenik veli (Fig. 1). It has an area of $58.20 \mathrm{~km}^{2}$ (Borovac, 2002: 356), it is $19 \mathrm{~km}$ long and 2-4.9 km wide (Prcić, 1990: 90). The western part of the island stretches in a W-E direction, and the eastern part in a NW-SE direction (Prcić, 1990: 90). It is mostly hilly with the highest peak Vela straža (237 m a.s.l.) near Gornje Selo village. The flattest part of the island is in the centre of the western part with a karstic polje $6 \mathrm{~km}$ long and up to $2.5 \mathrm{~km}$ wide. The coast is well indented and with many coves. The steepest, almost vertical, shores are on the southern part of the island, which is much like the southwestern shore exposed to the open sea. The nearest larger land is the island of Brač, only $700 \mathrm{~m}$ away, while the mainland is ca $10 \mathrm{~km}$ away (PrCIć, 1990: 90). Šolta was a part of a single mainland from which during geological history it was many times separated. During last 20.000 years the sea level rose ca 130 metres, the sea submerging valleys and plains, and what once were hills and mountains are now this island (Korbar \& MAUCH LenARDić, 2012: 187). On Šolta, Cretaceous limestones dominate and on them brown and red soils mixed with rocky grounds developed (Prсіć, 1990: 90; Borovac, 2002: 339, 341; Korbar \& Mauch Lenardić, 2012: 184). The climate is typically Mediterranean with mild and rainy winters and dry and hot summers. Mean yearly temperature is $16^{\circ} \mathrm{C}$, and mean precipitation is $847 \mathrm{~mm}$ (BEDALOv \& FISCHER, 1990: 105). It is situated in the sunniest part of Croatia with 2697 sunny hours per year, with a daily average of ca 7.4 hours (www.mzoip.hr). The most frequent winds are the bura, jugo and mistral. Vegetation is composed mainly of degraded stages of Mediterranean forest developed in the form of hard-leaved macchias of carob trees and wild olives (Borovac, 2002: 344), or in the form of garrigues and rocky pastures and dry grasslands (Bedalov \& Fischer, 1990: 105). There are planted forests of Aleppo pine (Pinus halepensis) (Bedalov \& Fischer, 1990: 106). On Šolta ca $40 \%$ of the surface is covered with forests, $42 \%$ with pastures, $11 \%$ is cultivated, a bit more than $6 \%$ is abandoned or unfertile ground (Sušić et al., 1990: 107). Regarding cultures, olive groves prevail, vineyards are mostly untended, and vegetable gardening is modest and not even sufficient for people's own needs (Sušıć et al., 1990: 107; Elezović, 2012; Kaštelanac, 2012).

The island of Drvenik veli is situated $3.4 \mathrm{~km}$ NW from Šolta, and $1.8 \mathrm{~km}$ away from the mainland (Fig. 1). It has a surface area of $12.07 \mathrm{~km}^{2}$ (Borovac, 2002: 356) which is almost 5 times less than Šolta. The highest peak is Buhalj at $178 \mathrm{~m}$ a.s.l. (hr.wikipedia.org). 


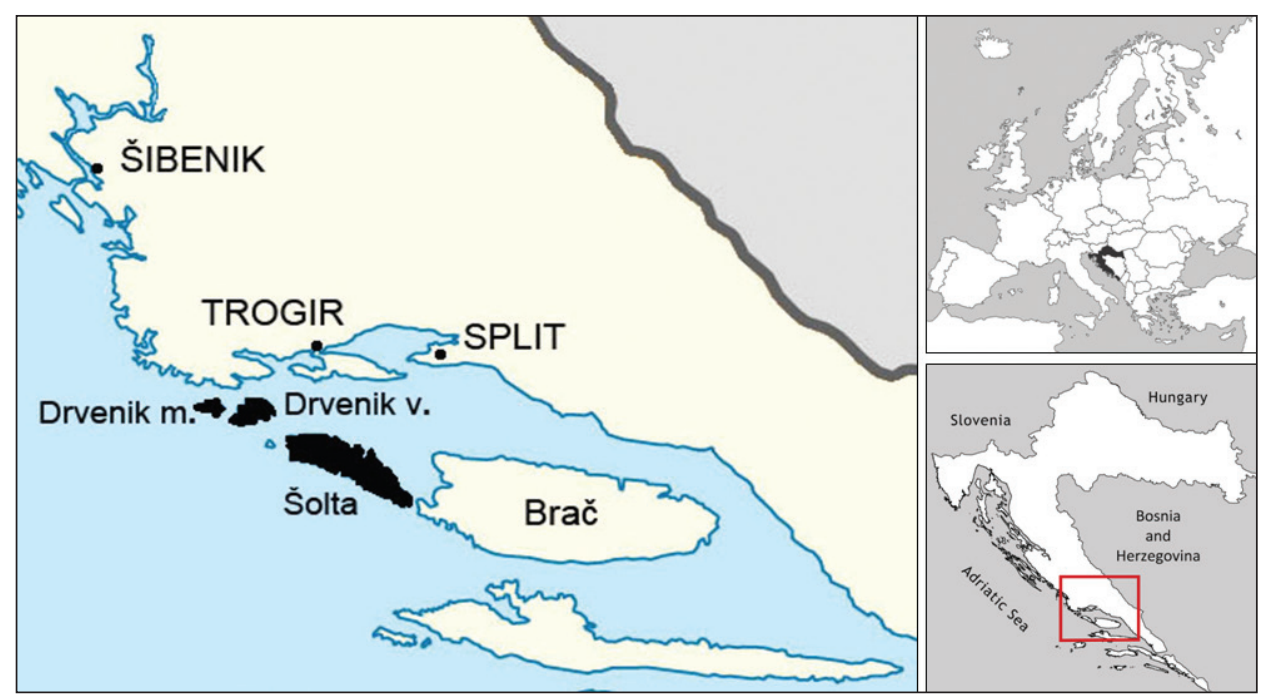

Fig. 1. Position of the researched islands in Croatia.

The island of Drvenik mali is $1.5 \mathrm{~km} \mathrm{~W}$ from Drvenik veli, and $1.9 \mathrm{~km}$ away from the mainland (Fig. 1). It has an area of $3,30 \mathrm{~km}^{2}$ (Borovac, 2002: 356) or three times smaller than Drvenik veli. The highest peak is Glavica with $79 \mathrm{~m}$ a.s.l. (hr.wikipedia.org).

The climatic, geological and pedological characteristics of Drvenik veli and Drvenik mali are similar to those on Šolta (Borovac, 2002: 339, 341-343). Climazonal vegetation on Drvenik veli and Drvenik mali is different than that on Šolta, and is made up of the evergreen holm oak forest (Borovac, 2002: 344). On these islands, as well as on almost all Croatian Adriatic islands, this forest grows only in degraded stages in the form of macchias, garrigues and rocky pastures. The few inhabitants grow olives and carob trees (hr.wikipedia.org).

\section{The history of research}

The oldest published data about land snails of the three islands originate from 1842 and apply to Drvenik veli. The then famous German malacologist and entomologist H. C. Küster described his voyage along the eastern Adriatic coast in 1840 in 1841 and noted two taxa for Drvenik veli (KüsTER, 1842). Other works from the 19th century also speak about land malacofauna of this island only (Brusina, 1866; Küster, 1870; Boettger, 1882). It was large enough to be interesting to naturalists and much nearer to the mainland than the larger Šolta, which at that time made Drvenik veli suitable for organizing short surveys from the mainland. Still, there were only a few surveys, and in the scarce papers only parts of results were published. Only taxa that were unexpected or interesting in some other way are mentioned, which led to only 4 land snail species being recorded during the entire 19th century. In the 20th century from papers about particular genera or species (Berberović, 1963, 1964; Nordsieck, 1969) we find out that on Drvenik veli there are 3 species more. If we exclude papers that cite published data (FrANK, 1991, 2000), these papers are still the only ones that speak about the existence of those 3 species of land snails on Drvenik veli. In the 21st century we find out that Lindholmiola 
corcyrensis (Rossmässler, 1836), mentioned for Drvenik veli as early as in 1842 (KüsTER, 1842) and relatively recently (SUBAI \& NEUBERT, 2014), in fact does not live there (ŠTAMOL, 2016). Thus according to the literature data on Drvenik veli there are 7 land snail species, two taxa are disputed, and one remains unidentified („,eine neue Clausilia”, KüsTER, 1842). We can conclude that although the first data about land snails on Drvenik veli were published as early as 1842 , which is only 16 years after the first data about Croatian island land malacofauna (ŠтAмоL et al., 2012a), its malacofauna remained fairly unknown until today, either as a consequence of the lack of systematic research, or the lack of published (complete) results of research that was conducted in this way.

The first data about the land snails of Drvenik mali were published only in the 20th century in systematic papers about one species or genus (BERBERović, 1964; NordsIECK, 1969). The latter author is cited by FrAnk $(1991,2000)$. In the 21st century there are neither new nor cited published data. Few papers and authors the low interest among naturalists in its malacofauna, in consequence of the small size of this island. Either research was not systematic, or the results were not (completely) published, or there results were published in systematic papers where only certain genera or species were elaborated, and it all led to the fact that today we know only two land snail species on Drvenik mali, which clearly shows the weakness of knowledge about its malacofauna.

The first land snails of Šolta were recorded no earlier than the mid-20th century. It was then that JAECKEL \& MeIsE (1956) published malacological results of the zoological and botanical research that was from September 11 to October 1, 1954, undertaken in Yugoslavia and Albania by the University of Hamburg. We do not know how long the research lasted on Šolta or on which area, but according to the results (only 3 recorded species) we can definitely conclude that malacological research of this island was only superficial. The following data about land snails of Šolta are still not results of a systematic research of its malacofauna, but only of research into certain species or genera (Berberović, 1962, 1963, 1964, 1967; Nordsieck, 1969; SubAi, 1980; Štamol \& Jovanović, 1990) or a consequence of citing the published data (FrANK, 1991, 2000). So at the end of the 20th century on Šolta there were only 9 species of land snails known. In the 21st century, thanks to the naturalistic curiosity and publicistic activity of the Šolta inhabitant Dinko Sule, 3 species and 1 genus more were recorded for the island (SulE, 2012, 2012a, 2013, 2014). We conclude that so far 13 land snail species with shells are known to live on Šolta, and that such a small number is a consequence of the poorly conducted faunistic research or of the lack of complete published results of systematic research.

\section{MATERIALS AND METHODS}

Field research in Šolta, Drvenik veli and Drvenik mali was done by Vesna Štamol and Eduard Kletečki from the Croatian Natural History Museum in Zagreb in April of 1998 and by Zoltán Péter Erőss from the Hungarian Natural History Museum in July of 2011 and in August of 2016. In the research we took care that all the main macrohabitats and microhabitats were included, and that localities were distributed through the entire island area and in all vertical zones as evenly as possible. Larger species were hand collected, and for collecting smaller taxa we took samples of soil and litter from which snails were isolated after drying and sifting through sieves of decreasing mesh size. A single locality would be researched into for about half an hour. Slugs were not a part of this research due to different collecting methods. The material is stored in the General Collection of Recent Molluscs in the Croatian Natural History Museum in Zagreb and in the collection of second author and a part of the material stored in Mollusca Depar- 
tment of the Hungarian Natural History Museum in Budapest. Data acquired from the identification of the shells collected by D. Sule in the area of Grohote were added to our results for the site 18 .

For determining the islands' biodiversity we used the Whittaker index of biodiversity $\left(\mathrm{I}_{\mathrm{W}}\right)$ and the maximum index of biodiversity $\left(\mathrm{I}_{\max }\right)$, which are quotients of total species number and average species number per site $\left(\mathrm{I}_{\mathrm{W}}\right)$, i.e. total species number and maximum species number on a site of a certain area $\left(\mathrm{I}_{\text {max }}\right)$ (CAMERon \& PoKryszKo, 2005), in this case of an island.

\section{RESULTS AND DISCUSSION}

\section{A list of sites}

Each locality in the list is marked by a number whose position is shown in Fig. 2 (for Šolta island) or in Fig. 3 (for Drvenik veli and Drvenik mali islands). Then follow to-

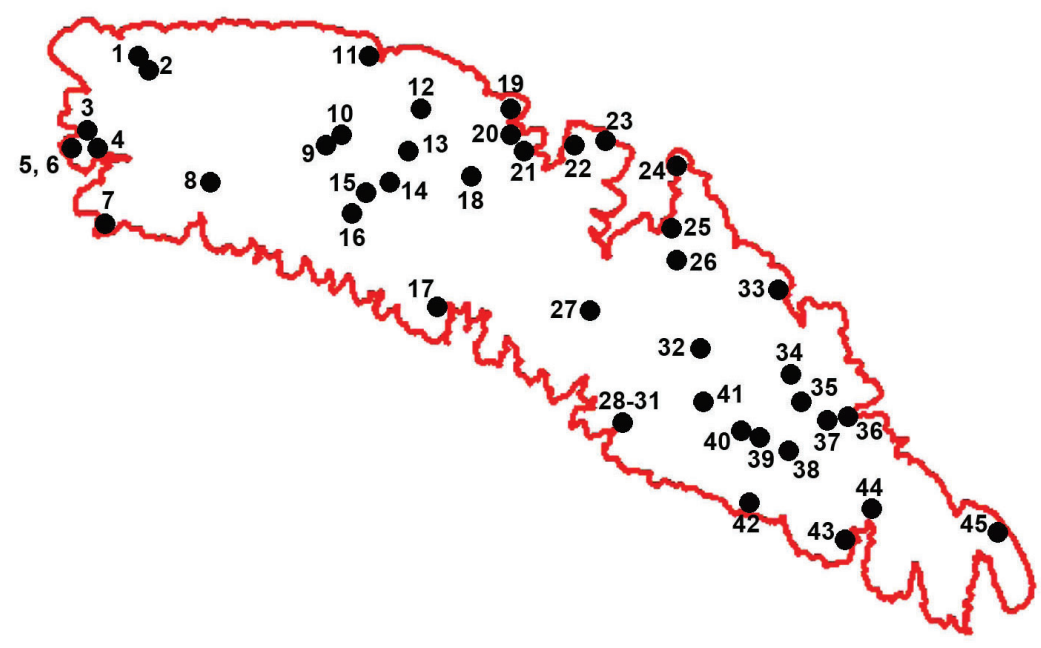

Fig. 2. The position of study localities on Šolta island. Numbers in the figure correspond to the numbers in the list of sites.
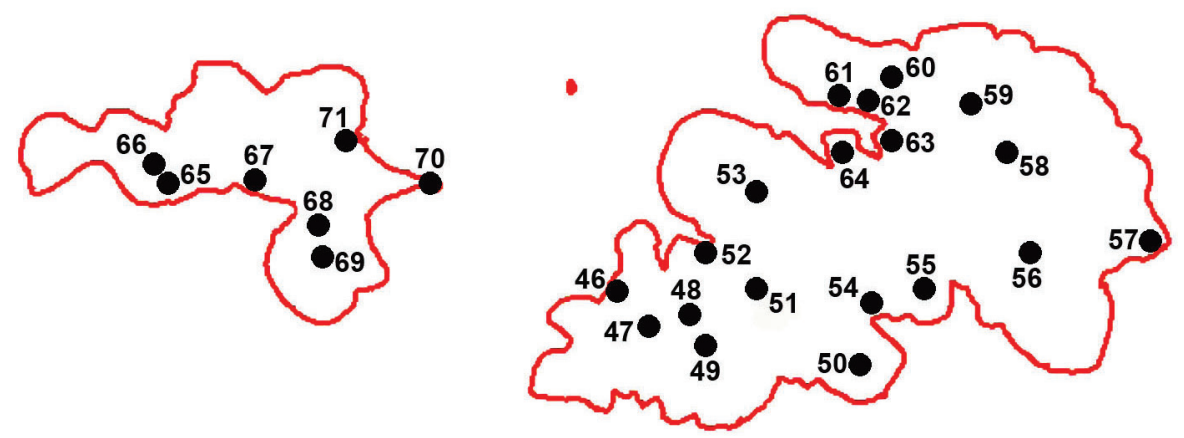

Fig. 3. The position of study localities on Drvenik veli and Drvenik mali islands. Numbers in the figure correspond to the numbers in the list of sites. 
ponyms given from larger to smaller, altitude, UTM designation, GPS coordinates and habitat. GPS coordinates were not taken using a GPS device during the field survey, but were subsequently determined from the 1:25000 topographic maps of the MiLitary GEOGRAPHY INSTITUTE (1984), and are given in the WGS84 coordinate system. Toponyms were taken also from the mentioned topographic maps 1:25000. Locality numbers 1-45 apply to Šolta, 46-64 to Drvenik veli, and 65-71 to Drvenik mali.

1. Šolta (island), Maslinica (village) - 1.8 km NNE, Vela Stiniva (bay)-0.4 km SE; $110 \mathrm{~m}$ a.s.1.; WJ90; $43.4094^{\circ} \mathrm{N}, 16.2181^{\circ} \mathrm{E}$; dry stone walls.

2. Šolta (island), Maslinica (village) - $1.75 \mathrm{~km} \mathrm{NE}$, Vela Stiniva (bay)-0.7 km SE; $130 \mathrm{~m}$ a.s.l.; WJ90; $43.4074^{\circ} \mathrm{N}, 16.2209^{\circ} \mathrm{E}$; stone house.

3. Šolta (island), Maslinica (village); $5 \mathrm{~m}$ a.s.l.; WJ90; $43.3984^{\circ} \mathrm{N}, 16.2084^{\circ} \mathrm{E}$; gardens, near houses.

4. Šolta (island), Maslinica (village) - 2.2 km SE, Šešula (bay); 5 m a.s.1.; WJ90; $43.3946^{\circ} \mathrm{N}, 16.21087^{\circ} \mathrm{E}$; rocks by the sea, garrigue.

4A. Šolta (island), Maslinica (village) - 2.2 km SE, Šešula (bay); $5 \mathrm{~m}$ a.s.l.; WJ90; $43.3948^{\circ} \mathrm{N}, 16.2099^{\circ} \mathrm{E}$; rocks in pine grove.

5. Šolta (island), Maslinica (village) - $0.3 \mathrm{~km}$ SSW, Sv. Nikola (church; cemetery); $45 \mathrm{~m}$ a.s.l.; WJ90; $43.3957^{\circ} \mathrm{N}, 16.2056^{\circ} \mathrm{E}$.

6. Šolta (island), Maslinica (village) $-0.5 \mathrm{~km}$ SW; $4 \mathrm{~m}$ a.s.1.; WJ90; $43.3959^{\circ} \mathrm{N}$, $16.2031^{\circ} \mathrm{E}$; pine grove.

7. Šolta (island), Maslinica (village) - 1.4 km SSE, Marinča rat (cape); $10-20 \mathrm{~m}$ a.s.l.; WJ90; $43.3836^{\circ} \mathrm{N}, 16.2109^{\circ} \mathrm{E}$; dry stone walls.

8. Šolta (island), Maslinica (village) - 1.4 km SSE, Poganička glava (cape); 100 m a.s.l.; WJ90; $43.3897^{\circ} \mathrm{N}, 16.2334^{\circ} \mathrm{E}$; olive grove.

9. Šolta (island), Donje Selo (village) - $0.25 \mathrm{~km} \mathrm{SW,} \mathrm{Sv.} \mathrm{Jelena} \mathrm{(chapel);} 105 \mathrm{~m}$ a.s.l.; $\mathrm{XJ00} ; 43.3974^{\circ} \mathrm{N}, 16.2587^{\circ} \mathrm{E}$; dry stone walls.

10. Šolta (island), Donje Selo (village); $110 \mathrm{~m}$ a.s.l.; XJ00; $43.3986^{\circ} \mathrm{N}, 16.2610^{\circ} \mathrm{E}$; church.

11. Šolta (island), Donje Selo (village) - 1.25 km NNE, Donja Krušica (bay); 2-10 m a.s.1.; XJ00; $43.4094^{\circ} \mathrm{N}, 16.2667^{\circ} \mathrm{E}$; rocks, pine grove.

12. Šolta (island), Srednje Selo (village) - $0.6 \mathrm{~km} \mathrm{~N}$, Strličina (summit); $180 \mathrm{~m}$ a.s.l.; $\mathrm{XJ00} ; 43.4023^{\circ} \mathrm{N}, 16.2772^{\circ} \mathrm{E}$; rocks.

13. Šolta (island), Srednje Selo (village); $120 \mathrm{~m}$ a.s.l.; XJ00; $43.3971^{\circ} \mathrm{N}, 16.2761^{\circ} \mathrm{E}$; church, dry stone wall.

14. Šolta (island), Srednje Selo (village) $-0.75 \mathrm{~km} \mathrm{SW}$, Srednje polje (field), Basilija (pool); $100 \mathrm{~m}$ a.s.l.; XJ00; $43.3917^{\circ} \mathrm{N}, 16.2689^{\circ} \mathrm{E}$; at pool.

15. Šolta (island), Donje Selo (village) - 1 km SSE, Sv. Mihovil (church); $104 \mathrm{~m}$ a.s.l.; $\mathrm{XJ00} ; 43.3905^{\circ} \mathrm{N}, 16.2643^{\circ} \mathrm{E}$; dry stone walls.

16. Šolta (island), Grohote (village) - $2.1 \mathrm{~km}$ SWW, Vela straža (summit); $210 \mathrm{~m}$ a.s.l.; $\mathrm{XJ} 00 ; 43.3868^{\circ} \mathrm{N}, 16.2617^{\circ} \mathrm{E}$; rocks.

17. Šolta (island), Grohote (village) - 2.25 km SSW, Tatinja (bay); 20-30 m a.s.l.; XJ00; $43.3720^{\circ} \mathrm{N}, 16.2807^{\circ} \mathrm{E}$; rocks.

18. Šolta (island), Grohote (village); $120 \mathrm{~m}$ a.s.l.; XJ00; $43.3921^{\circ} \mathrm{N}, 16.2878^{\circ} \mathrm{E}$; church.

19. Šolta (island), Rogač (village) - 0.7 km NNW, Gradina (region); 70 m a.s.l.; $43.4014^{\circ} \mathrm{N}, 16.2937^{\circ} \mathrm{E}$; rocks.

20. Šolta (island), Rogač (village) - 0.5 km NNW, Kašjun (bay); 10-20 m a.s.1.; XJ00; $43.3989^{\circ} \mathrm{N}, 16.2934^{\circ} \mathrm{E}$; pine grove, rocks.

21. Šolta (island), Rogač (village); 5 m a.s.l.; XJ00; $43.3955^{\circ} \mathrm{N}, 16.2973^{\circ} \mathrm{E}$; church. 
22. Šolta (island), Rogač (village) - 0.7 km NEE, Žustova (bay); 20 m a.s.l.; XJ00; $43.3964^{\circ} \mathrm{N}, 16.3056^{\circ} \mathrm{E}$; rocks.

23. Šolta (island), Rogač (village) - 1.4 km NEE, Timun (region), Tepli bok (bay); $10 \mathrm{~m}$ a.s.l.; XJ00; $43.3969^{\circ} \mathrm{N}, 16.3131^{\circ} \mathrm{E}$; stony grassland.

24. Šolta (island), Nečujam (bay), Bok od rata (region); $5 \mathrm{~m}$ a.s.1.; XJ00; 43.3930 $\mathrm{N}$, $16.3260^{\circ} \mathrm{E}$; stone walls.

25. Šolta (island), Nečujam (village); $5 \mathrm{~m}$ a.s.l.; $\mathrm{XJ} 00 ; 43.3841^{\circ} \mathrm{N}, 16.3252^{\circ} \mathrm{E}$; stone walls.

26. Šolta (island), Nečujam (village) - $0.7 \mathrm{~km} \mathrm{S;} 60 \mathrm{~m}$ a.s.l.; XJ00; $43.3784^{\circ} \mathrm{N}, 16.3248^{\circ} \mathrm{E}$; rocks, cliffs.

27. Šolta (island), Gornje Selo (village) - 3,25 km NW, Rudine (region); $110 \mathrm{~m}$ a.s.l.; $\mathrm{XJ00} ; 43.3720^{\circ} \mathrm{N}, 16.3090^{\circ} \mathrm{E}$; olive grove, rocks.

28. Šolta (island), Gornje Selo (village) - $2.1 \mathrm{~km} \mathrm{~W}$, Grabova bay (bay); 2-10 m a.s.l.; $\mathrm{XJ} 00 ; 43.3541^{\circ} \mathrm{N}, 16.3154^{\circ} \mathrm{E}$; rocks.

29. Šolta (island), Gornje Selo (village) - $2.1 \mathrm{~km} \mathrm{~W}$, Grabova uvala (bay); $40 \mathrm{~m}$ a.s.l.; $\mathrm{XJ} 00 ; 43.3543^{\circ} \mathrm{N}, 16.3181^{\circ} \mathrm{E}$; rocks.

30. Šolta (island), Gornje Selo (village) - $2.1 \mathrm{~km} \mathrm{W,} \mathrm{Grabova} \mathrm{uvala} \mathrm{(bay);} 60 \mathrm{~m}$ a.s.l.; $\mathrm{XJ} 00 ; 43.3544^{\circ} \mathrm{N}, 16.3194^{\circ} \mathrm{E}$; rocks.

31. Šolta (island), Gornje Selo (village) - $2 \mathrm{~km} \mathrm{W,} \mathrm{Grabova} \mathrm{uvala} \mathrm{(bay);} 70 \mathrm{~m}$ a.s.1.; XJ00; $43.3545^{\circ} \mathrm{N}, 16.3201^{\circ} \mathrm{E}$; rocks.

32. Šolta (island), Gornje Selo (village) - $1.4 \mathrm{~km} \mathrm{NNW,} \mathrm{Koludrovi} \mathrm{doci} \mathrm{(region);} 130 \mathrm{~m}$ a.s.l.; $\mathrm{XJ} 00 ; 43.3655^{\circ} \mathrm{N}, 16.3339^{\circ} \mathrm{E}$; pit.

33. Šolta (island), Stomorska (village) $-0.55 \mathrm{~km} \mathrm{NNW;} 5 \mathrm{~m}$ a.s.1.; XJ00; $43.3741^{\circ} \mathrm{N}$, $16.3493^{\circ} \mathrm{E}$; rocks.

34. Šolta (island), Gornje Selo (village) - 1 km NNE, Gospa od Stomorina (church); 175 $\mathrm{m}$ a.s.1.; XJ00; $43.3609^{\circ} \mathrm{N}, 16.3496^{\circ} \mathrm{E}$; along walls.

35. Šolta (island), Gornje Selo (village) - 0.9 km NE, Vela straža (summit); 235 m a.s.l.; $\mathrm{XJ} 00 ; 43.3580^{\circ} \mathrm{N}, 16.3521^{\circ} \mathrm{E}$; pit.

36. Šolta (island), Gornje Selo (village) - 1.75 km NEE, Gornja Krušica (bay); XJ10; 2 m a.s.l.; $43.3566^{\circ} \mathrm{N}, 16.3631^{\circ} \mathrm{E}$; dry stone walls, meadow.

37. Šolta (island), Gornje Selo (village) - 1.5 km NEE, Gornja Krušica (hamlet); $50 \mathrm{~m}$ a.s.l.; XJ00; $43.3561^{\circ} \mathrm{N}, 16.3596^{\circ} \mathrm{E}$; pine grove, garrigue, rocks.

38. Šolta (island), Gornje Selo (village) - $0.8 \mathrm{~km} \mathrm{SE;} 155 \mathrm{~m}$ a.s.l.; XJ00; $43.3491^{\circ} \mathrm{N}$, $16.3509^{\circ} \mathrm{E}$; pit.

39. Šolta (island), Gornje Selo (village) - $0.25 \mathrm{~km} \mathrm{S;} 150 \mathrm{~m}$ a.s.l.; XJ00; $43.3515^{\circ} \mathrm{N}$, $16.3424^{\circ} \mathrm{E}$; dry stone walls.

40. Šolta (island), Gornje Selo (village) - $0.25 \mathrm{~km} \mathrm{W;} 150 \mathrm{~m}$ a.s.l.; XJ00; 43.3537º N, $16.3378^{\circ} \mathrm{E}$; at pool.

41. Šolta (island), Gornje Selo (village) - 0.8 km NW, Srednji dolac (pool); $140 \mathrm{~m}$ a.s.l.; $\mathrm{XJ} 00 ; 43.3593^{\circ} \mathrm{N}, 16.3343^{\circ} \mathrm{E}$; at pool.

42. Šolta (island), Gornje Selo (village) - $1.4 \mathrm{~km} \mathrm{S,} \mathrm{Vela} \mathrm{ploča} \mathrm{(region);} 50 \mathrm{~m}$ a.s.l.; XJ00; $43.3422^{\circ} \mathrm{N}, 16.3406^{\circ} \mathrm{E}$; rocks.

43. Šolta (island), Gornje Selo (village) - 2.5 km SSE, Stračinska uvala (bay); 20 m a.s.l.; $\mathrm{XH} 19 ; 43.3379^{\circ} \mathrm{N}, 16.3634^{\circ} \mathrm{E}$; rocks.

44. Šolta (island), Gornje Selo (village) - 2.5 km SE, Stračinska uvala (bay); XJ10; $10 \mathrm{~m}$ a.s.1.; WH19; $43.3411^{\circ} \mathrm{N}, 16.3663^{\circ} \mathrm{E}$; rocks, stony grassland.

45. Šolta (island), Gornje Selo (village) - 4,5 km SE, Bad (region); 20 m a.s.1.; XH19; $43.3389^{\circ} \mathrm{N}, 16.3935^{\circ} \mathrm{E}$; garrigue.

46. Drvenik veli (island), Širan (hamlet) - $300 \mathrm{~m}$ NW, Kale (bay); 0-5 m a.s.1.; WJ91; $43.4376^{\circ} \mathrm{N}, 16.1165^{\circ} \mathrm{E}$; stony grassland. 
47. Drvenik veli (island), Širan (hamlet); $35 \mathrm{~m}$ a.s.1.; WJ91; $43.4357^{\circ} \mathrm{N}, 16.1192^{\circ} \mathrm{E}$; stone houses.

48. Drvenik veli (island), Komorovica (summit) - $400 \mathrm{~m}$ NNW; $45 \mathrm{~m}$ a.s.l.; WJ91; $43.4364^{\circ} \mathrm{N}, 16.1249^{\circ} \mathrm{E}$; rocks, pine grove.

49. Drvenik veli (island), Komorovica (summit); $84 \mathrm{~m}$ a.s.1.; WJ90; $43.4330^{\circ} \mathrm{N}$, $16.1264^{\circ} \mathrm{E}$; garrigue, stony ground.

50. Drvenik veli (island), Kačine (hamlet), Starica (pool); $15 \mathrm{~m}$ a.s.l.; WJ90; $43.4315^{\circ} \mathrm{N}$, $16.1443^{\circ} \mathrm{E}$; rocks.

51. Drvenik veli (island), Kačine (hamlet); $50 \mathrm{~m}$ a.s.1.; WJ91; $43.4378^{\circ} \mathrm{N}, 16.1339^{\circ} \mathrm{E}$; dry stone walls.

52. Drvenik veli (island), Mali Porat (hamlet), Mala luka (bay); 1-5 m a.s.l.; WJ91; $43.4415^{\circ} \mathrm{N}, 16.1269^{\circ} \mathrm{E}$; rocks, grassland.

53. Drvenik veli (island), Drvenik Veli (village) - $1.4 \mathrm{~km} \mathrm{SW,} \mathrm{Sv.} \mathrm{Nikola} \mathrm{(church);} 94 \mathrm{~m}$ a.s.l.; WJ91; $43.4456^{\circ} \mathrm{N}, 16.1341^{\circ} \mathrm{E}$; rocks.

54. Drvenik veli (island), Kokosinje (hamlet), Solinska uvala (bay); 10 m a.s.l.; WJ91; $43.4366^{\circ} \mathrm{N}, 16.1448^{\circ} \mathrm{E}$; stony grassland.

55. Drvenik veli (island), Kokosinje (hamlet); 30-40 $\mathrm{m}$ a.s.1.; WJ91; $43.4391^{\circ} \mathrm{N}, 16.1507^{\circ} \mathrm{E}$; dry stone walls.

56. Drvenik veli (island), Krknjasi (hamlet); 85 m a.s.l.; WJ91; $43.4406^{\circ} \mathrm{N}, 16.1615^{\circ} \mathrm{E}$; dry stone walls.

57. Drvenik veli (island), Krknjasi (hamlet), Krknjaš (beach); 0-10 m a.s.l.; WJ91; $43.4412^{\circ} \mathrm{N}, 16.1744^{\circ} \mathrm{E}$; dry stone walls.

58. Drvenik veli (island), Drvenik Veli (village) - 0.9 km SEE, Buhaj (summit); 160-178 m a.s.l.; WJ91; $43.4479^{\circ} \mathrm{N}, 16.1577^{\circ} \mathrm{E}$; rocks.

59. Drvenik veli (island), Drvenik Veli (village) - 0.6 km NEE, Brda (hamlet); $110 \mathrm{~m}$ a.s.l.; WJ91; $43.4518^{\circ} \mathrm{N}, 16.1535^{\circ} \mathrm{E}$; stone houses.

60. Drvenik veli (island), Drvenik Veli (village) - $0.4 \mathrm{~km} \mathrm{~N}$, Bobovišće (hamlet); $96 \mathrm{~m}$ a.s.l.; WJ91; $43.4548^{\circ} \mathrm{N}, 16.1469^{\circ} \mathrm{E}$; stony grassland.

61. Drvenik veli (island), Drvenik Veli (village) - $0.5 \mathrm{~km} \mathrm{NW,} \mathrm{Drvenik} \mathrm{veli} \mathrm{(haven);}$ 20-30 $\mathrm{m}$ a.s.l.; WJ91; $43.4527^{\circ} \mathrm{N}, 16.1410^{\circ} \mathrm{E}$; rocks.

62. Drvenik veli (island), Drvenik Veli (village) - $0.3 \mathrm{~km} \mathrm{NW,} \mathrm{Drvenik} \mathrm{veli} \mathrm{(haven);} 5 \mathrm{~m}$ a.s.1.; WJ91; $43.4519^{\circ} \mathrm{N}, 16.1441^{\circ} \mathrm{E}$; stony grassland.

63. Drvenik veli (island), Drvenik Veli (village); $12 \mathrm{~m}$ a.s.l.; WJ91; $43.4503^{\circ} \mathrm{N}, 16.1465^{\circ} \mathrm{E}$; stone walls of the church; grassland.

64. Drvenik veli (island), Drvenik Veli (village) - $0.5 \mathrm{~km} \mathrm{SW}$, Grabule (region); $20 \mathrm{~m}$ a.s.l.; WJ91; $43.4485^{\circ} \mathrm{N}, 16.1407^{\circ} \mathrm{E}$; dry stone walls.

65. Drvenik mali (island), Glavica (hill); $60 \mathrm{~m}$ a.s.1.; WJ81; $43.4462^{\circ} \mathrm{N}, 16.0671^{\circ} \mathrm{E}$; rocks.

66. Drvenik mali (island), Glavica (hill); $79 \mathrm{~m}$ a.s.l.; WJ81; $43.4471^{\circ} \mathrm{N}, 16.0668^{\circ} \mathrm{E}$; rocks.

67. Drvenik mali (island), Vela Rina (hamlet); $5 \mathrm{~m}$ a.s.1.; WJ81; $43.4464^{\circ} \mathrm{N}, 16.0775^{\circ} \mathrm{E}$; rocks, grassland.

68. Drvenik mali (island), Petomavar (hamlet); $20 \mathrm{~m}$ a.s.l.; WJ81; $43.4428^{\circ} \mathrm{N}, 16.0848^{\circ} \mathrm{E}$; dry stone walls.

69. Drvenik mali (island), Petomavar (hamlet); $40 \mathrm{~m}$ a.s.l.; WJ81; $43.4407^{\circ} \mathrm{N}, 16.0848^{\circ} \mathrm{E}$; dry stone walls, grassland.

70. Drvenik mali (island), Kuknara (cape), Kuknara (hamlet); WJ81; 5-8 m a.s.l.; $43.4464^{\circ} \mathrm{N}, 16.0972^{\circ} \mathrm{E}$; rocks, garrigue.

71. Drvenik mali (island), Borak (hamlet); $1-10 \mathrm{~m}$ a.s.l.; WJ81; $43.4498^{\circ} \mathrm{N}, 16.0885^{\circ} \mathrm{E}$; dry stone walls, rocks. 


\section{List of terrestrial snails with finding sites}

The list gives names of species and subspecies found during our field research and recorded in the literature for the investigated islands. Taxa are listed in order and nomenclature is given according to ŠTAMoL (2010). In front of the species and subspecies name there is an ordinal number, except in the cases when we consider it is not present on the researched islands. After the valid name follow taxon names $(\mathrm{N})$ used in the literature which gives finding localities on the researched islands, field work localities (F) marked by number from the list of sites and from Figures 2 and 3, and finding localities from the literature (L). Field work localities from Šlta are given in black numbers, Drvenik veli in red, and Drvenik mali in blue. A locality marked by a question mark means that specimens from the respective locality could not be identified with certainty, often because they were juvenile or badly preserved. A question mark in front of the species name means that the taxon could not be identified with certainty in the research area in general. Localities from the literature were given separately for each island. If we considered that a literature toponym could cause a dilemma while being defined, we cited it.

\section{Cochlostoma (Cochlostoma) scalarinum scalarinum (A. \& B. J. Villa, 1841)}

N: Cochlostoma scalarinum (Villa): Berberović, 1962: 48;

Cochlostoma sp.: Sule, 2014: 61.

F: 1, 2, 3, 4, 4A, 5, 6, 7, 8, 11, 12, 14, 15, 16, 17, 18, 19, 20, 21, 22, 23, 26, 27, 28, 29, 30, 31, $32,33,34,35,37,38,39,41,42,43,44,45,46,47,48,49,50,51,52,53,54,55,56,57,58,59$, $60,61,62,63,65,66,67,69,70,71$.

L: island Šolta: Šolta (island) (Berberović, 1962: 48); Grohote (village), Podvelagomila (part of the village) (Sule, 2014: 61).

Remark: The latest genus revision (ZALLot et al., 2015) placed C. scalarinum into Wagneriola subgenus.

\section{Pomatias elegans (O. F. Müller, 1774)}

N: Pomatias elegans (O. F. Müller, 1774): ŠTAmol \& Jovanović, 1990: 15;

Pomatia elegans: Sule, 2012: 34;

Pomatias elegans: Sule, 2012a: 306, 2014: 61.

F: 3, 4, 4A, 5, 6, 7, 9, 10, 11, 12, 13, 15, 16, 17, 18, 19, 20, 21, 22, 23, 24, 25, 26, 27, 28, 32, $33,34,36,37,39,42,43,44,45,46,47,48,49,50,51,52,53,54,55,56,57,58,59,60,61,62$, $63,64,65,66,68,69,70,71$.

L: island Šolta: Šolta (island) (Sule, 2012: 34, 2012a: 306); Maslinica (ŠTAmol \& JovANOvić, 1990: 15; Sule, 2014: 61 [citing Štamol \& Jovanović, 1990: 15, author's note]).

\section{Cecilioides (Cecilioides) acicula (O. F. Müller, 1774)}

F: $1,2,18 ?, 35,49,57,61$.

\section{Cecilioides (Cecilioides) veneta (Strobel, 1855)}

F: $14,18,19,25 ?, 31,39,51,56,58,60 ?$.

\section{Cecilioides sp.}

F: $4,16,18,27,43,50,65,69$. 


\section{Rumina decollata (Linnaeus, 1758)}

N: Rumina decollata: Sule, 2012: 34, 2012a: 306, 2014: 61.

F: 3, 5, 18, 40 .

L: island Šolta: Šolta (island) (Sule, 2012: 34, 2012a: 306, 2014: 61).

\section{Delima (Delima) blanda conspurcata (Rossmässler, 1836)}

F: $13,47,51,55,59,69$.

\section{Delima (Piceata) piceata (Rossmässler, 1836)}

N: Clausilia piceata Ziegler: BrusinA, 1866: 115.

L: island Drvenik veli: Drvenik veli (island) (Brusina, 1866: 115: „Zirona“)

Remark: in the list of the malacological collection of Carl Kutschig, BRUsinA (1866: 115) emphasizes that Kutschig himself doubted this species existed on Drvenik veli. Delima piceata is an endemic snail of Italy (BANK, 2007, 2010, 2013), i.e. central Italy (WELTER-SCHultes, 2012: 308).

\section{Delima (Semirugata) hiltrudis H. Nordsieck, 1969}

F: $4,17,18,19,22,28,29,31,39,42,43,44$.

\section{Delima (Semirugata) semirugata semirugata (Rossmässler, 1836)}

N: Delima (Semirugata) semirugata (Rossmässler): NordsIEcK, 1969: 273;

Delima semirugata (Rossmaessler 1836): FrANK, 1991: 362;

Delima (Semirugata) semirugata (Rossmaessler 1836): FrANK, 2000: 95.

F: 1, 2, 3, 4, 4A, 5, 6, 7, 8, 9, 10, 11, 12, 13, 15, 16, 17, 19, 20, 21, 23, 25, 26, 27, 28, 33, 34, 35, 36, 37, 38, 41, 43, 44, 45, 46, 48, 49, 50, 52, 53, 54, 55, 56, 57, 58, 65, 66, 67, 70.

L: island Šolta: Šolta (island) (NoRDSIECK, 1969: 273; FRANK, 1991: 362, 2000: 95 [FRANK, 1991, 2000 citing NordsIEck, 1969, author's note]).

Island Drvenik veli: Drvenik veli (island) (NordsIEck, 1969: 273, FrANK, 2000: 95: „Inseln Drveniki“; Frank, 1991: 362: „I. Drveniki“. [FranK, 1991, 2000 citing Nordsieck, 1969, author's note]).

Island Drvenik mali: Drvenik mali (island) (NoRDSIECK, 1969: 273, FrANK, 2000: 95: „Inseln Drveniki“; Frank, 1991: 362: „I. Drveniki“ [Frank, 1991, 2000 citing Nordsieck, 1969, author's note]).

\section{Delima (Semirugata) vidovichii robusta (Küster, 1847)}

N: Clausilia (Delima) robusta K. mut. albina Bttg.: BoetTger, 1882: 40;

Delima (Delima) robusta K.: WAGNER, 1924: 110.

F: 46, 47, 48, 50, 51, 52, 53, 54, 55, 56, 57, 58, 59, 60, 61, 62, 63, 64, 68, 69, 71.

L: island Drvenik veli: Drvenik veli (island) (Boettger, 1882: 40: „Insel Zirona“; WAGNER, 1924: 110: "Zirona“).

\section{Delima sp.}

F: $7,13,18,24,33,43,47,50,51,55,56,57,59,61,62,65,66,68,69$.

\section{Hypnophila pupaeformis (Cantraine, 1836)}

F: $2,3,4,5,6,8,9,10,13,15,16,17,18,19,20,22,25,26,27,28,29,31,32,34,36,37$, $39,40,42,43,44,47,48,50,51,53,54,55,57,58,59,61,62,64,65,66,68,69,71$. 
11. Chondrula quinquedentata quinquedentata (Rossmässler, 1837)

F: 7, 8, 12, 19, 25, 26, 28, 30, 37, 44, 62, 65.

\section{Chondrula sp.}

F: 71.

Pseudochondrula seductilis (Rossmässler, 1837)

N: Bulimus (Chondrula) seductilis Ziegler: Küster, 1870: 89;

Imparietula seductilis (Rossmässler 1837): Fischer et al., 2000: 54.

L: island Drvenik veli: Drvenik veli (island) (Küster, 1870: 89: „Zirona grande“; FisCHER et al., 2000: 54 „I. Zirona grande“. [FISCHER et al., 2000 citing KüsTER, 1870, author's note]).

\section{Mediterranea hydatina hydatina (Rossmässler, 1838)}

F: $25,59,62$.

\section{3. ? Oxychilus (Oxychilus) draparnaudi (H. Beck, 1837)}

F: $3,5,25,57$.

Oxychilus sensu lato sp.

F: 6 .

\section{Vitrea botterii (L. Pfeiffer, 1853)}

F: $11,15,26,30,32,33,35,36,37,46,47,52,53,58,62,65,66,71$.

\section{Vitrea subrimata (Reinhardt, 1871)}

F: 2, 3? , 4, 4A, 6, 7, 8, 13, 16, 17, 18, 19, 20, 22, 24, 25, 28, 31, 32, 33, 34, 35, 37, 38, 42, 43, $44,45,48,50,52,54,55,56 ?, 57,58,59,60,61,62 ?$.

\section{Vitrea sp.}

F: 4 A.

16. Chilostoma (Liburnica) setosa setosa (A. Férussac, 1832)

N: Liburnica setosa: Sule, 2014: 61.

F: 17, 18, 26, 28, 29, 31, 39, 42, 43.

L: island Šolta: Grohote (village), Kavčina (part of the village) (Sule, 2014: 61).

\section{Cantareus apertus (Born, 1778)}

N: Helix naticoides: Sule, 2012: 34, 2012a: 306, 2014: 61.

F: 3, 18, 25.

L: island Šolta: Šolta (island) (SulE, 2012: 34, 2012a: 306, 2014: 61).

18. Cornu aspersum aspersum (O. F. Müller, 1774)

N: Helix aspersa Müll.: Berberović, 1964: 6-9;

Helix aspersa: Sule, 2012: 34, 2012a: 306, 2014: 61.

F: 10, 13, 18, 46, 47, 51, 56, 59, 62, 63, 64, 68, 69, 70, 71.

L: island Šolta: Šolta (island) (BERBERović, 1964: 6-9; Sule, 2012: 34, 2012a: 306, 2014: 61).

island Drvenik veli: Drvenik veli (island) (BERBERović, 1964: 6-9).

island Drvenik mali: Drvenik mali (island) (Berberović, 1964: 6-9). 


\section{Eobania vermiculata vermiculata (O. F. Müller, 1774)}

N: Eobania vermiculata (Müll.): Berberović, 1962: 48, 1963;

Eobania vermiculata: Berberović, 1967: 18; Sule, 2012: 34, 2012a: 306, 2014: 61.

Eobania vermiculata (Müller, 1774): RAĐA et al., 2012.

F: $2,3,4,5,9,10,11,13,14,15,17,18,20,21,23,24,25,28,33,34,36,39,40,41,42,43$, $45,46,49,50,51,53,54,55,56,57,59,60,62,63,64,66,67,70,71$.

L: island Šolta: Šolta (island) (BERBERović, 1963: 50, 71, 1967: 18: Sl. 5; RAĐA et al., 2012: 299-300, 302-305; Sule, 2012: 34, 2012a: 306, 2014: 61); Maslinica (village) (BERBERović, 1963: 5, 15, 17, 19, 21, 23, 25, 28); Nečujam (village) (Berberović, 1963: 5, 15, 17, 19, 21 , 23, 25, 28); Stomorska (village) (Berberović, 1962:48, 1963: 5, 15, 17, 19, 21, 23, 25, 28).

island Drvenik veli: Drvenik veli (island) (BERBERović, 1963: 5, 15, 17, 19, 21, 23, 25, 28).

\section{Lindholmiola corcyrensis (Rossmässler, 1838)}

N: Helix contorta: Küster, 1842: 301;

Remark: Helix contorta can be synonymized with two now valid taxa, with Lindholmiola corcyrensis (Rossmässler, 1838) and with Vitrea diaphana (S. Studer, 1820), i.e. for Croatian territory with $V$. d. erjaveci (Brusina, 1870). We assume that in the cited paper (KüSTER, 1842) the data relates to Lindholmiola corcyrensis, because it is a bigger snail, which probably-could be spotted by a researcher (Küster) during a short visit to the island.

Lindholmiola corcyrensis (Rossmässler, 1838): Subai \& Neubert, 2014: 46.

L: island Drvenik veli: Drvenik veli (island) (Küster, 1842: 301: „Insel Zirona grande“; Subai \& Neubert, 2014: 46: „,,Dalmatien, Zirona Grande“ ").

Remark: as explained in ŠтAмоL (2016) Lindholmiola corcyrensis does not inhabit either of the investigated Dalmatian islands, or Croatia at all. Both data (Küster, 1842: 301; SubAi \& Neubert, 2014: 46) have their origins in the material collected in the 19th century, probably from specimens washed up by the sea. A check made by Dr Janssen in the Senckenberg Museum Collection did not confirm the suspicion about specimens of Lindholmiola corcyrensis from the Senckenberg Museum Collection, studied in the paper by Subai \& Neubert (2014) being collected by Küster, so it could not be confirmed that the specimens that KüsTER (1842: 201) wrote of are the same as those later written about by Subai \& Neubert (2014). This implies that the washing up of this species on the shores of Drvenik veli is not an isolated case. L. corcyrensis has been washed up on other parts of Croatian coast and islands, from the southernmost parts to the northern Dalmatia, as explained in detail by ŠTAMOL (2016).

\section{Cernuella (Cernuella) cisalpina cisalpina (Rossmässler, 1837)}

F: 3, 5, 9, 10, 13, 18, 20, 25, 39, 40, 52, 62, 63.

Cernuella (Cernuella) virgata (Da Costa, 1778)

N: Helicella variabilis (Draparnaud): JAECKEL \& MeISE, 1956: 26.

L: island Šolta: Šolta (island) (JAECKel \& MeIse, 1956: 26).

\section{Cernuella sp.}

N: Cernuella sp.: Sule, 2014: 61.

L: island Šolta: Rogač (village), Banje (bay) (Sule, 2014: 61).

\section{Hiltrudia mathildae (Westerlund, 1881)}

F: 12, 17, 19, 20, 21, 46, 47, 48, 50, 51, 52, 53, 55, 56, 57, 58, 59, 60, 61, 62, 65, 66, 69, 71. 


\section{Monacha (Monacha) cartusiana (O. F. Müller, 1774)}

N: Monacha carthusiana (O. F. Müller): JAEcKEL \& Meise, 1956: 26.

F: $2,3,5,9,10,11,12,15,17,18,20,22,23,24,25,26,27,32,34,36,40,41,45,46,47$, $48,49,50,51,52,53,55,56,59,62,63,64,67,68,69,71$.

L: island Šolta: Šolta (island) (JAECKEL \& MeISE, 1956: 26).

Remark: Sule (2014: 61) writes about the very probable specimens of Monacha cartusiana in the area of Grohote on the Šolta island ("Monacha cartusiana vrlo vjerojatno"). Because of the expressed doubt, we can not take the cited information into account.

\section{Monacha (Monacha) parumcincta (Menke, 1828)}

F: $1,3,4 \mathrm{~A}, 6,7,8,11,12,14,15,16,17,19,22,24,25,26,28,29,30,33,34,35,36,37,38$, $39,40,42,43,44,45,49,56,58,60,61,62,65$.

Remark: Sule (2014: 61) writes about very probable specimens of Monacha parumcincta in the area of Grohote on the Šolta island ("Monacha parumcincta vrlo vjerojatno“). Because of the expressed doubt, we can not take the cited information into account.

\section{Monacha sp.}

N: Monacha sp.: Sule, 2014: 61.

F: $12,15,18,21,56,64$.

L: island Šolta: Grohote (village), Podvelagomila (part of the village) (SuLE, 2014: 61). Helicidae sensu lato

F: $13,17,37,54,57,61,62,64,65,69,71$.

\section{Punctum (Punctum) pygmaeum (Draparnaud, 1801)}

F: $4,6,7,11,15,16,26,28,30,31,32,33,35,36,37,39,42,46,48,52,57,58,60$.

\section{Granaria illyrica (Rossmässler, 1835)}

N: Abida frumentum (Draparnaud): JAECKEL \& Meise, 1956: 22.

F: 4, 4A, 6, 7, 11, 17, 19, 20, 22, 24, 25, 26, 28, 30, 33, 37, 42?, 43, 44, 45, 49, 61, 65, 66, 67, 70.

L: island Šolta: Šolta (island) (JAECKEL \& MeISE, 1956: 22).

Remark: According to FeHÉr et al. (2010) G. illyrica is a subspecies of G. frumentum (Draparnaud, 1801).

\section{Granopupa granum (Draparnaud, 1801)}

F: 28,42 .

\section{Rupestrella philippii philippii (Cantraine, 1840)}

F: 4, 17, 19, 22, 26, 28, 29, 30, 31, 33, 37, 42, 43, 61, 62, 63.

\section{Rupestrella rhodia (Roth, 1839)}

F: $4,12,26,30,31,35,37,42,44,50,53,54,55,56,58,59,60,62,65,68,69$.

\section{Lauria (Lauria) cylindracea (Da Costa, 1778)}

F: $3,5,6,20,39,46,47,48,51,52,55,56,59,65,68,69$.

30. Odontocyclas kokeilii (Rossmässler, 1837)

F: 22, 51, 55, 59, 64. 


\section{Pyramidula rupestris (Draparnaud, 1801) s.l.}

F: 47, 48, 51, 53, 54, 55, 56, 57, 59, 68, 69.

Remark: Former Pyramidula rupestris, considered a widely distributed European species, has been divided into 4 species based on the morphological characteristics of the shell (Gittenberger \& BANK, 1996), and 3 of those species were considered to live in Croatia (ŠTAMOL, 2010: 38). Newer research (RAZKIN et al., 2017) showed that identification of Pyramidula based only on conciological characteristics is mostly not possible, so we leave specimens from our researched islands undefined, as Pyramidula rupestris s.l.

\section{Acanthinula aculeata (O. F. Müller, 1774)}

F: $16,24,25,26,28,29,30,31,32,33,34,35,36,37$.

\section{Truncatellina callicratis (Scacchi, 1833)}

F: 2, 3, 4A, 6, 7, 11, 14, 15, 16, 17, 19, 20, 21, 22, 23, 26, 27, 28, 30, 31, 32, 33, 34, 35, 36, $37,39,41,42,44,46,47,48,49,50,51,52,53,54,55,56,57,58,61,63,65,68,69$.

\section{Truncatellina cylindrica (A. Férussac, 1807)}

F: $4,30,31$.

\section{Truncatellina sp.}

F: $17,30$.

\section{Poiretia cornea (Brumati, 1838)}

N: Poiretia algira (Brug.): Berberović, 1962: 48;

Poiretia algira: Berberović, 1967: 18/S1. 5;

Poiretia cornea (Brumati 1838): Subai, 1980: 160/161;

Poiretia cornea: Sule, 2013: 6/7.

F: $1,3,5,7,8,10,11,12,13,14,15,16,17,18,19,20,21,22,24,25,26,27,28,31,32,33$, $34,35,36,37,38,39,40,42,43,44,46,47,48,50,51,53,55,56,57,58,59,60,61,62,63,64$, $65,66,68,69,70,71$.

L: island Šolta: Šolta (island) (BERBERović, 1962: 48, 1967: 18/Sl. 5.); Stomorska (village) (Berberović, 1962: 48); Rogač (village) (Subai, 1980: 160/161); Grohote (village) (Sule, 2013: 6/7).

\section{Aegopis acies (A. Férussac, 1832)}

$\mathrm{N}$ : Aegopis sp.: Sule, 2014: 61.

F: $3,9,10,13,18,46,47,48,51,54,55,56,57,59,62,63,64,69$.

L: island Šolta: Grohote (village), Podvelagomila (part of the village) (Sule, 2014: 61).

In the field research on the island of Šolta 45 localities were investigated and 34 land snail species were found there. In the literature 14 species were recorded. One of them, Cernuella (Cernuella) virgata, was not found in our research. This is possibly caused by the only local presence C. (C.) virgata on Šolta. In the literature less than $50 \%$ of taxa found in our field work was recorded. Among taxa not registered in the literature those smaller than $5 \mathrm{~mm}$ prevail. The reason is that most of the Solta literature deals with species or genera that belong to big snails (BERBERović, 1962, 1963, 1964, 1967; NordsiecK, 1969; Subai, 1980; ŠTAmol \& Jovanović, 1990; RaĐA et al., 2012). Other literature cite data 
from the above mentioned papers (FrANK, 1991, 2000; Sule, 2014), so it is again about bigger snails or it brings results from one's own research which were, apparently, based on individual sampling of snails, when again specimens of bigger species are found (JAeCKel \& Meise, 1956; Sule, 2012, 2012a, 2013, 2014). So there is a lack of papers based on systematic island research. Of taxa found on Šolta we definitely have to emphasize i) Delima (Semirugata) hiltrudis, until now endemic for the nearby island of Brač; it is interesting that already while describing this taxon, Nordsieck (1969) anticipated its existence on Šolta; ii) Odontocyclas kokeilii, a snail relatively widely distributed in Croatia, but rare to find, this being its second recorded island finding locality (the first island finding locality in Croatia is Brač island (ŠTAMoL, 1986)).

During our field work on 19 localities on the island of Drvenik veli we found 29 land snail species. In the literature 7 species were recorded, all of them having shells bigger than $5 \mathrm{~mm}$, and belonging to medium big and big land snails. Small species were not recorded. The reasons are similar to those in the case of Šolta: i) papers deal with certain species or genera belonging to bigger snails (Boettger, 1882; WAGNer, 1924; Nordsieck, 1969; Berberović, 1963, 1964; Subai \& Neubert, 2014); ii) paper is a kind of a travel diary which has no intention to list the entire fauna but only the 'interesting' taxa (KüstER, 1842); iii) on the island there has been no systematic research or else no papers have been published with complete results of such investigations (Küster, 1870); iv) in other papers, data from the above mentioned works are cited (FIsCHER et al., 2000; FrANK, 2000). Our field work confirmed 4 species, and 3 species were not found. We consider two of those three (Lindholmiola corcyrensis, Delima (P.) piceta) not to exist on Drvenik veli, and not in Croatia either (BANK, 2007, 2010, 2013; ŠTAMOL, 2016). The third species from the literature, Pseudochondrula seductilis, judging by its distribution range and habitat, could inhabit Drvenik veli. Our not finding it is possibly a consequence of it being only locally present on the island. It is possible that the population of this grassland species diminished or disappeared. Namely, the abundance of grassland land snail species in Europe is decreasing because of habitat loss, and according to the observations of Fischer et al. (2000: 54) the same is happening in Croatia. Still, to apply this fact to Drvenik veli we should have more reliable old data, and complete the research by investigating target habitats on Drvenik veli. In the malacofauna of Drvenik veli an interesting taxon is i) Delima (S.) vidovichii robusta, recorded on many Dalmatian islands from Lastovo and Kopište in the south (WITTE, 1965: 212, 214) to Babina guzica (Kornati archipelago) in the north (ŠTAmoL et al., 2012), but non-existing on the nearby Šolta and Brač (Š́tAmoL, 1986; KitTeL, 2002); ii) Odontocyclas kokeilii, this being its third recorded island finding locality in Croatia.

On the island of Drvenik mali 20 species of land snails were found on 7 researched localities. In the literature two species were recorded (3\%). Both were confirmed by our field work, and belong to big land snails. There being only a few recorded species is a consequence of the type of papers that publish the data. These are papers that i) deal with a genus presented on the island with only one species (NorDSIECK, 1969); ii) deal with only one species (BERBERović, 1964); iii) cite published data (FrANK, 1991, 2000). For Drvenik mali, as for Šolta and Drvenik veli, we can conclude that systematic research into land malacofauna, as well as the published results of such research, are deficient. As on Drvenik veli, on Drvenik mali there is Delima (S.) vidovichii robusta, as mentioned, a taxon of wider distribution in Dalmatia, but non-existing on nearby Šolta and Brač. Comparing Delima genus on three investigated islands (Solta, Drvenik veli, Drvenik mali) and on the nearby island of Brač (ŠтAмоL, 1986; Kittel, 2002) it is interesting that on each island there are three representatives. They have two taxa, Delima (D.) blanda conspurcata and D. (S.) semirugata semirugata, in common, while D. (S.) hiltrudis inhabits 
only Brač and Šolta, and D. (S.) vidovichii robusta only Drvenik veli and Drvenik mali. Of unexpected results we should mention the existence of Pyramidula rupestris s.l. on Drvenik veli and Drvenik mali, and its non-existence on Šolta. P. rupestris s.l. has suitable habitats on Šlta; maybe the fact that we did not find it shows its localized presence, or our insufficient systematic research of suitable habitats. From the expected results we emphasize the fact that all snail species missing on Drvenik veli, in comparison to Šolta, are missing also on Drvenik mali. Also all species existing on Drvenik mali, exist on Drvenik veli. The most common taxa on the investigated islands inhabit stony grasslands, smaller rocks and cracks (Cochlostoma (C.) scalarinum scalarinum, Pomatias elegans, Delima (S.) semirugata semirugata, D. (S.) vidovichii robusta, Hypnophila pupaeformis). Similar results were shown in other research we have conducted on Croatian islands (STAMOL, 1986, 2004, 2013; ŠTAmol \& VelKovrh, 1995; Štamol \& KleteČKI, 2005; Štamol et al., 2012). Stony grasslands dominated by stones are the species-poorest habitats, while moderately anthropogenic habitats prove to be the richest in land snail species.

Biodiversity measures taking into account the number of species of an area and the biodiversity of researched localities, the Whittaker $\left(\mathrm{I}_{\mathrm{W}}\right)$ and the maximum $\left(\mathrm{I}_{\max }\right)$ biodiversity indices, are calculated for investigated islands and compared to those of nearby Brač island (ŠтAмоl et al., 2014) (Tab. 1). Both indices showed that the biggest island, Brač, has the highest biodiversity, followed by islands that diminish in the order: Šolta, Drvenik veli, and the smallest Drvenik mali. These results are not surprising because it is one of the oldest ecological laws that diversity increases with surface area. $\mathrm{I}_{\mathrm{W}}$ and $\mathrm{I}_{\max }$ differ in ratios of the highest and lowest diversity. While in the Whittaker index it is 1.83:1, in the maximum index it is 1.78:1. A smaller ratio of $I_{\max }$ is a consequence of the cancelling of a something found frequently, that localities in a certain area with few species are inhabited by the impoverished fauna of richer localities, where the poorer localities are not inhabited by species living elsewhere (CAMEron \& Poкryszко, 2005), which makes biodiversity results according to $\mathrm{I}_{\max }$ more reliable. In Tab. 1, beside the mentioned indices $\mathrm{I}_{\mathrm{W}}$ and $\mathrm{I}_{\max }$ there are islands' areas, number of investigated localities, and average species number per locality $\left(S_{L}\right)$. Drvenik veli has the highest $S_{L}$. On Drvenik veli there are two localities with 18 species, and even 3 localities with 17 species. On the five times bigger Šolta, with twice as many localities researched than on Drvenik veli, there is no locality with 18 species, and three of them have 17 species as well. We think such a high average number per locality on Drvenik veli is a consequence of the diverse habitats on this island, and especially of the not very large human influence, shown in the moderate urbanization of the coast and of entire island, in the comparative lack of asphalted roads and motor vehicles. If we wish to maintain such a good condition of malacofauna, we should preserve the still relatively intact nature of Drvenik veli.

On Šolta and Drvenik mali we should also try to at least preserve the present conditions. That means we should prevent the disappearance or reduction of suitable habitats for land snails, which occur by i) excessive building of housing and tourist facilities, ii) abandonment of the traditional way of building houses, dry walls and roads, iii) abandonment of the old ways of planting olive groves and vineyards, iv) depopulation (abandonment) of old settlements, reducing the availability of suitable (moderately) anthropogenic habitats, which in the Mediterranean parts of Croatia are shown to be the richest in snail species, v) the reduction of cattle breeding, which leads to pastures being overgrown and the disappearance of grassland species. To preserve the malacofauna of the investigated islands it is important, along the above mentioned facts, especially to protect at least some localities and their habitats with endemic species, e.g. Delima (S.) hiltrudis on Šolta, or those with rare species, e.g. Odontocyclas kokeilii. 
Tab. 1. Island area (A), number of researched localities per island (L), species number on island $\left(\mathrm{S}_{\mathrm{O}}\right)$, average species number per site $\left(\mathrm{S}_{\mathrm{L}}\right)$, maximum species number per site $\left(\mathrm{S}_{\mathrm{M}}\right)$, the Whittaker biodiversity index $\left(\mathrm{I}_{\mathrm{W}}\right)$, the maximum biodiversity index $\left(\mathrm{I}_{\max }\right)$ for the researched islands. Data for Brač are taken from ŠтAмоL et al. (2014).

\begin{tabular}{|l|c|c|c|c|c|c|c|}
\hline island & $\mathrm{A}\left(\mathrm{km}^{2}\right)$ & $\mathrm{L}$ & $\mathrm{S}_{\mathrm{O}}$ & $\mathrm{S}_{\mathrm{L}}$ & $\mathrm{S}_{\mathrm{M}}$ & $\mathrm{I}_{\mathrm{W}}$ & $\mathrm{I}_{\max }$ \\
\hline Brač & 394.57 & 25 & 43 & 11.00 & 20 & 3.91 & 2.15 \\
\hline Šolta & 58.20 & 45 & 34 & 10.19 & 17 & 3.34 & 2.00 \\
\hline Drvenik veli & 12.07 & 19 & 29 & 13.00 & 18 & 2.23 & 1.61 \\
\hline Drvenik mali & 3.30 & 7 & 20 & 9.37 & 17 & 2.13 & 1.18 \\
\hline
\end{tabular}

\section{CONCLUSION}

In our research of land snail species with shells on central Dalmatian islands of Šolta, Drvenik veli and Drvenik mali (Croatia) it was shown that the biggest of them, Šolta, has 34 species, mid-sized Drvenik veli has 29 species, and the smallest of them, Drvenik mali, has 20 species. Among them we point out Odontocyclas kokeilii, Šolta and Drvenik veli being its second i.e. third island finding localities in Croatia, and the endemic Deli$m a(S$.) hiltrudis, known only from the island of Brač, now found on Šolta, and D. (S.) vidovichii robusta found on Drvenik veli and on Drvenik mali. In the available literature only $41 \%$ of the existing malacofauna of Šolta was recorded, $17 \%$ in the case of Drvenik veli, and only $3 \%$ in the case of Drvenik mali, which shows that on the investigated islands until now no systematic research has been conducted. Lindholmiola corcyrensis and Delima (P.) piceata, recorded in the literature, do not inhabit Drvenik veli, and the data were either the consequence of material washed up by the sea, or of mistakes in the identification or labelling of the material. We consider our failure to find species from the literature such as Cernuella (C.) virgata for Šolta and Pseudochondrula seductilis for Drvenik veli was the consequence of the localized appearance of these snails or their extinction in these islands. Biodiversity values, i.e. the Whittaker biodiversity index $\left(\mathrm{I}_{\mathrm{W}}\right)$ and the maximum biodiversity index $\left(\mathrm{I}_{\max }\right)$, are higher on the bigger islands, as expected. The average species number per locality is the highest on Drvenik veli, which is a consequence of the preserved habitats and the moderate human influence with the traditional way of life preserved. Land malacofauna on Šolta, Drvenik veli and Drvenik mali should be preserved at least at the present stage, which could be achieved by limiting urbanization, especially in the coastal area, and by maintaining the traditional way of building houses, agriculture and cattle breeding.

\section{Acknowledgements}

We thank Dr Ronald Janssen (Germany) for data about Lindholmiola corcyrensis deposited in the Collection of the Senckenberg Museum in Frankfurt am Main, Hartmut Nordsieck (Germany) for help in identifying the genus Delima, Dr Benjamín Gómez Moliner (Spain) for discussion about the genus Pyramidula, and Dinko Sule (Grohote, Croatia) for giving us his material from Šolta and for numerous information about the Šolta fauna and literature. 


\section{REFERENCES}

BAnk, R. A. (ed.), 2007: Mollusca, Gastropoda. Fauna Europaea version, 1.3, http://www.faunaeur.org/.

BANK, R. A., 2010: Fauna europaea project. Checklist of species-group taxa of the continental Mollusca (excl. Bivalvia) living in Albania and former Yugoslavia, Manuscript, updated by Ruud A. Bank, 2512-2010.

BANK, R. A. (ed.), 2013: Mollusca, Gastropoda. Fauna Europaea version, 2.6.2, http://www.faunaeur.org/.

Bedalov, M. \& Fischer, M. A., 1990: Basic features of plant overgrowth on the Island of Šolta. p. 103-107. In: Minovilović, M. A. (ed.): Otok Šolta: Monografija. 280 pp. Mihovilović, Zagreb. [in Croatian]

Berberović, Lj., 1962: A contribution to the knowledge of the biology of the predaceous snail Poiretia algira (Brug.). Arhiv biol. nauka 14 (1/2), 47-49. [in Bosnian]

Berberović, LJ., 1963: Microevolution in the species Eobania vermiculata (Müll.) on the Yugoslav MiddleAdriatic shore and adjacent Islands. Godišnjak Biol. Instit. Univerz. Sarajevo 16, 3-76. [in Bosnian]

Berberović, LJ., 1964: Die neue unterart Helix aspersa ssp. pelagosana von der Insel Palagruža im Adriatischen Meere. Godišnjak Biol. Instit. Univerz. Sarajevo 17, 3-11. [in Bosnian]

Berberović, LJ., 1967: Predators of species Eobania vermiculata (Müll.) and Helix aspersa Müll. Ekologija 2 (1/2), 15-21. [in Bosnian]

Boettger [Böttger], O., 1882: Liste der bis jetzt bekannten Deviationen und albinen und flavinen Mutationen des Gehäuses bei der Gattung Clausilia Drap. Nachrichtsbl. Deutsch. Malakozool. Ges. 14 (3), $36-43$.

Bole, J., 1958: Zoogeographische Übersicht der Molluskenfauna der Insel Krk. Biol. vestn. 6, 118-123. [In Slovenian]

Borovac, I., 2002: Veliki atlas Hrvatske. 480 pp. Mozaik knjiga, Zagreb.

Brusina, S., 1866: Contribuzione pella Fauna dei molluschi dalmati. Verh. k. k. zool. bot. Ges. Wien 16, Beilage, 1-134.

Cameron, R. A. D. \& Pokryszko, B. M., 2005: Estimating the species richness and composition of land mollusc communities: problems, consequences and practical advice. J. Conchol. 38, 529-547.

Duplančić Leder, T., Ujević, T. \& Čala, M., 2004: Coastline lenghts and areas of islands in the Croatian part of the Adriatic sea determined from the topographic maps at the scale of $1: 25000$. Geoadria 9 (1), 5-32.

Elezović, D., 2012: Poljoprivreda Šolte. p. 373-383. In: RADMAN, Z. (ed.): Otok Šolta, monografija. 631 pp. Šolta Municipality, Grohote.

Fehér Z., Deli T. \& Sólymos, P., 2010: Revision of Granaria frumentum (Draparnaud 1801) (Mollusca, Gastropoda, Chondrinidae) subspecies occurring in the eastern part of the species' range. J. Conchol. 40, 201-217.

Fischer, W., Kittel, K., Reischütz, A. \& Reischütz, P. L., 2000: Ein Beitrag zur Kenntnis der Molluskenfauna von Pag (Nord-Dalmatien, Kroatien). Nachrichtenbl. Ersten Vorarlberger Malak. Ges. 8, 53-59.

FrANK, C., 1991: Über Molluskenfunde aus Jugoslawien und den der Küste vorgelagerten Inseln des adriatischen Meeres. Zeitschr. angew. Zool. 78 (3), 349-380.

Frank, C., 2000: Mollusca (Gastropoda et Bivalvia): eine historische Sammlung aus Hollabrunn (Niederösterreich). Wiss. Mitt. Niederösterr. Landesmuseum 13, 47-167.

Gittenberger, E. \& Bank, R. A., 1996: Anew start in Pyramidula (Gastropoda Pulmonata: Pyramidulidae). Basteria 60, 71-78.

JAeCKel, S. \& Meise, W., 1956: Über Land- und Süsswasserschnecken Jugoslawiens und Albaniens. Mitt. Hamburg. Zool. Mus. Inst. 54, 21-31.

KaštelanaC, V., 2012: Velike promjene u šoltanskoj poljoprivredi od 1990. do danas. p. 383-385. In: RADMAN, Z. (ed.): Otok Šolta, monografija. 631 pp. Šolta Municipality, Grohote.

Kittel, K., 2002: Ein Beitrag zur Kenntnis der Molluskenfauna der Insel Brač (Dalmatien, Kroatien). p. 429-448. In: Falkner, M., Grom, K., Speight, M. C. D. (eds.): Collectanea malacologica. Friedrich-Held Gesellschaft, Conchbooks Hackenheim.

Korbar, T. \& Mauch Lenardić, J., 2012: Kako je nastala Šolta - geološke značajke. p. 184- 187. In: RADMAN, Z. (ed.): Otok Šolta, monografija. 631 pp. Šolta Municipality, Grohote.

Küster, H. C., 1842: Reiseberichte aus Dalmatien und Montenegro. Isis von Oken, 1842 (4), $283-301$.

Küster, H. C., 1870: Die Binnenmollusken-Fauna von Triest, Istrien, Dalmatien und Montenegro. Bericht naturforsch. Ges. Bamberg 9, 86-101. 
Nordsieck, H., 1969: Zur Anatomie und Systematik der Clausilien, VII. Dinarische Clausiliidae, I: Das Genus Delima. Arch. Molluskenkunde 99 (5/6), 267-284.

Prcić, M., 1990: Geografske karakteristike otoka Šolte. p. 90-92. In: RADMAN, Z. (ed.): Otok Šolta, monografija. 631 pp. Šolta Municipality, Grohote.

RaĐA, B., RaĐA, T., Puizina, J., Šamanić, I. \& Šantić, M., 2012: Shell characteristics of land snail Eobania vermiculata (Müller, 1774) (Helicidae) from Croatia. Amer. Malac. Bull. 30 (2), 299-307.

Razkin, O., Gómez-Moliner, B. J., Vardinoyannis, K., Martínez-Ortí, A. \& Madeira, J. M., 2017: Species delimitation for cryptic species complexes: case study of Pyramidula (Gastropoda, Pulmonata). Zoologica Scripta 46 (1), 55-72.

Reischütz, A. \& Reischütz, P. L., 1999: Ein Beitrag zur Kenntnis der Molluskenfauna der Insel Vis (Mitteldalmatien, Kroatien). Nachrichtenbl. Ersten Vorarlberger Malak. Ges. 7, 35-39.

Reischüтz, A. \& Reischüтz, P. L., 2000: Ein Beitrag zur Kenntnis der Molluskenfauna der Insel Mljet (Dalmatien, Kroatien). Nachrichtenbl. Ersten Vorarlberger Malak. Ges. 8, 60-65.

Reischütz, A., Reischütz, P. L., Fischer, W., Fraberger, U. \& Kiennast, H., 2002: Ein Beitrag zur Kenntnis der Molluskenfauna von Korcula (Dalmatien, Kroatien). Nachrichtenbl. Ersten Vorarlberger Malak. Ges. 10, 52-58.

Subai, P., 1980: Revision der lebenden Arten der Gattung Poiretia (Gastropoda: Oleacinidae). Arch. Molluskenkunde 110 (1979), 151-172.

Subai, P. \& Neubert, E., 2014: Revision of the genus Lindholmiola Hesse, 1931 (Gastropoda: Pulmonata: Helicodontidae). Contrib. Nat. Hist. 23, 1-94.

Sule, D., 2012: Prilog poznavanju faune i flore otoka Šolte. Bašćina 21, 30-44.

SulE, D., 2012a: Iz životinjskog svijeta otoka Šolte. p. 304-306. In: RADMAN, Z. (ed.): Otok Šolta, monografija. 631 pp. Šolta Municipality, Grohote.

Sule, D., 2013: Prilog poznavanju faune i flore otoka Šolte. Bašćina 22, 5-16.

Sule, D., 2014: Zapisi o šoltanskoj biološkoj raznolikosti. Bašćina 23, 53-67.

Sušić, G., Pallaoro, A., Radović, D. \& Stipčević, M., 1990: Ptice otoka Šolte. p. 107-111. In: Minovilović, M. A. (ed.): Otok Šolta: Monografija. 280 pp. Mihovilović, Zagreb.

Šтамоl, V., 1986: Contribution to the study of land snails (Gastropoda) of the island of Brač and endargement of endemic species. Biosistematika 12 (1), 45-56. [in Croatian]

Štamol, V., 2004: Terrestrial snails (Mollusca: Gastropoda terrestria) of the Telašćica Nature Park (Dugi otok, Croatia). Nat. Croat. 13 (2), 95-113.

ŠтAmol, V., 2010: A list of the land snails (Mollusca: Gastropoda) of Croatia, with recommendations for their Croatian names. Nat. Croat. 19 (1), 1-76.

ŠTamol, V., 2013: Kopneni puževi (Gastropoda terrestria) otoka Silbe. p. 103-110. In: MužInIĆ, J., PURGER, J. J. (eds.): Otok Silba, prirodno i kulturno blago. 322 pp. Sveučilište u Zadru, Zadar.

ŠтамоL, V., 2016: Is Lindholmiola corcyrensis (Rossmässler, 1838) (Mollusca: Gastropoda) extinct in Croatia? Nat. Croat. 25 (1), 159-162.

Štamol, V., Cameron, R., KletečKi, E., Vuković, M. \&. Grgurev, M., 2014: Species/area and other relationships in land snails (Mollusca: Gastropoda terrestria) faunas of some Adriatic islands. Nat. Croat. 23 (2), 317-334.

Štamol, V. \& Jovanović, B., 1990: Distribution of Land Snails Pomatias elegans (O. F. Müller, 1774) and Pomatias rivulare (Eichwald, 1829) (Mollusca: Gastropoda) in Yugoslavia. Scopolia 21, 1-42.

ŠTAmol, V. \& KLETEČKI, E., 2005: Terrestrial snails (Mollusca: Gastropoda terrestria) of Dugi otok (Croatia). Nat. Croat. 14 (1), 1-28.

ŠTamol, V., KLetečKi, E. \& Vuković, M., 2012: A contribution to the knowledge of the terrestrial snails (Mollusca: Gastropoda terrestria) of Kornati National Park (Croatia). Nat. Croat. 21 (2), 427-454.

Štamol, V. \& Poje, M., 1998: The fossil and recent malacofauna of the island of Susak (Croatia) (Gastropoda: Prososbranchia, Basommatophora, Stylommatophora). Malak. Abh. Mus. Tierkd. Dresden 19 (1), 103-117.

ŠTamol, V. \& Velkovrh, F., 1995: Contribution to the knowledge of land snail fauna of the islands of Cres and Lošinj (Croatia) (Gastropoda: Prosobranchia, Basommatophora, Stylommatophora). Malak. Abh. Mus. Tierkd. Dresden 17 (20), 219-237.

ŠTAmol, V., Vuković, M. \& KLETEČKI, E., 2012a: Literature review of terrestrial snail research for several Croatian islands. Nat. Croat. 21 (1), 165-190. 
Topografske Karte [Topographic Maps], 1984: scale 1:25000, sheets Drvenik mali (124-1-1), Drvenik veli (124-1-2), Grohote (124-2-1), Stomorska (124-2-3), Milna (124-2-4). Vojnogeogr. inst., Rep. geod. uprava SR Hrvatske, Zagreb.

Wagner, A. [J.], 1924: Systematisches Verzeichnis der mir heute bekannten Arten und Formen der Clausiliiden. III. Annal. Zool. Mus. Pol. Hist. Nat. 3 (3-4), 99-126.

Welter-Schultes, F. W, 2012: European non-marine molluscs, a guide for species identification. A1-A3, 679, Q1-Q78 pp. Planet Poster Editions, Göttingen.

WitTe, G. R., 1965: Ergebnisse neuer biogeographischer Untersuchungen zur Verbreitung transadriatischer Faunen- und Floren-Elemente. Bonner Zoolog. Beiträge 16 (3/4), 165-248.

Zallot, E., Groenenberg, D. S. J., De Mattia, W., Fehér, Z. \& Gittenberger, E., 2015: Genera, subgenera and species of the Cochlostomatidae (Gastropoda, Caenogastropoda, Cochlostomatidae). Basteria 78 (4-6), 63-88.

hr.wikipedia.org/wiki/, last accessed 05.12.2016.

http://www.mzoip.hr/doc/elaborat_zastite_okolisa_36.pdf

\title{
SAŽETAK
}

\section{Kopneni puževi (Mollusca: Gastropoda) otoka Šolte, Drvenika velog i Drvenika malog (Hrvatska)}

\author{
V. Štamol, Z. P. Erőss, E. Kletečki \& M. Vuković
}

Istraživanjem kopnenih puževa s kućicom na srednjedalmatinskim otocima Šolti, Drveniku velom i Drveniku malom (Hrvatska) nađeno je 36 vrsta. Ustanovljeno je da najveći otok, Šolta, ima 34 vrste, srednje veliki Drvenik veli 29 vrsta, a najmanji, Drvenik mali 20 vrsta. Među njima ističu se Odontocyclas kokeilii, puž relativno širokog areala u Hrvatskoj, ali rijetke pojavnosti, kojem su Šolta i Drvenik veli drugo, odnosno treće otočno nalazište u Hrvatskoj, te endemična zaklopnica Delima (Semirugata) hiltrudis, poznata samo s otoka Brača, nađena sada na Šolti. Kao neočekivani rezultat navodimo nalaz Pyramidula rupestris s.l. na Drveniku velom i Drveniku malom, uz istovremeno nenalaženje na većoj i pogodnim staništima obdarenoj Šolti. Druga svojta nepostojeća na Šolti, a nađena i već ranije zabilježena za Drvenik veli i Drvenik mali je Delima (Semirugata) vidovichii robusta, što ukazuje na granice njenog areala. U dostupnoj je literaturi zabilježeno 41 \% postojeće malakofaune otoka Šolte, 17 \% Drvenika velog, a samo 3 \% Drvenika malog. Među zabilježenim puževima samo su velike ili srednje velike svojte, što je nastalo zbog i) nekorištenja metode uzimanja uzorka tla i stelje, ii) monografskog karaktera djela posvećenih velikim vrstama ili rodovima, odnosno zbog nesustavno provedenih terenskih istraživanja cjelokupne kopnene malakofaune. Literaturno zabilježene Lindholmiola corcyrensis i Delima (Piceata) piceata ne obitavaju na otoku Drveniku velom, a podaci su posljedica ili morem naplavljenih primjeraka ili pogreške u determinaciji ili etiketiranju materijala. Smatramo da je naše nenalaženje literaturno zabilježenih vrsta Cernuella (Cernuella) virgata za Šoltu i Pseudochondrula seductilis za Drvenik mali rezultat lokalizirane pojavnosti ovih puževa ili njihovog izumiranja na navedenim otocima. Mjere biološke raznolikosti Whittakerov indeks biodiverziteta $\left(\mathrm{I}_{\mathrm{W}}\right)$ i maksimalni indeks biodiverziteta $\left(\mathrm{I}_{\max }\right)$ su očekivano veći na većim otocima. Prosječan broj vrsta po lokalitetu je najveći na Drveniku velom, što proizlazi iz očuvanosti staništa i održanja umjerenog utjecaja čovjeka s njegovanim tradicijskim načinom života. Kopnenu malakofaunu je potrebno na Šolti, Drveniku velom i Drveniku malom očuvati barem u sadašnjem stanju, što će se moći ostvariti ograničenjem urbanizacije, očuvanjem tradicijskog načina gradnje, poljoprivrede i stočarstva. 